\title{
COMPETENCIAS MEDIÁTICAS EN JÓVENES UNIVERSITARIOS. ANÁLISIS DE SABERES PARA PRODUCIR CONTENIDO DIGITAL EN UNA IES MEXICANA
}

\section{MEDIA COMPETENCE IN UNIVERSITY STUDENTS. ANALYSIS OF KNOWLEDGE OF PRODUCTION OF DIGITAL CONTENT IN A MEXICAN UNIVERSITY}

\author{
Abel Antonio Grijalva Verdugo, abel.grijalva@udo.mx; iD 0000-0001-8828-7269 \\ José Alejandro Lara Rivera; alejandro.lara@udo.mx; iD 0000-0001-9269-8132
}

Universidad Autónoma de Occidente (México)

\section{Resumen}

Esta investigación explora los niveles de competencia mediática (CM) de jóvenes universitarios para la creación y manipulación de contenido digital; saberes necesarios en una era altamente tecnologizada. El estudio se realiza en una universidad mexicana (UAdeO) a una muestra probabilística aleatoria simple compuesta por 346 estudiantes divididos por áreas de conocimiento. Se ejecutaron pruebas no paramétricas (Chi-Cuadrada) para identificar posibles diferencias estadísticas a razón de la variable de cruce. Los hallazgos dan cuenta de niveles de CM diferenciados intergrupos, además, si bien los jóvenes muestran saberes elevados en la producción de contenido digital, aún existen deficiencias en el manejo de bases de datos y programas informáticos especializados, por lo que sería relevante implementar estrategias pedagógicas que incrementen dichos conocimientos.

Palabras clave: Alfabetización mediática; Educación mediática; Educación Superior; Instrucción multimedia.

\section{Abstract}

This research explores the levels of media competence (MC) of university students for the creation and manipulation of digital content; knowledge needed in a highly technological era. The study is carried out in a Mexican university (UAdeO) to a simple random probabilistic sample composed of 346 students divided by areas of knowledge. Non-parametric tests (Chisquare) were performed to identify possible statistical differences on the basis of the crossover variable. The findings show differentiated levels of MC intergroups, in addition, although students show high knowledge in the production of digital content, there are still deficiencies in the management of databases and specialized computer programs, so it would be relevant to implement pedagogical strategies that increase such knowledge.

Keywords: Media Literacy; Media Education; Higher Education; Multimedia Instruction. 


\section{INTRODUCCIÓN}

La investigación forma parte de un estudio más amplio titulado "evaluación de saberes digitales para estudiantes de educación superior" que tiene como objetivos conocer los niveles de competencia mediática y digital en jóvenes universitarios. Se partió del interés de analizar las posibles relaciones entre el currículum escolar y la categoría creación y manipulación de contenido digital, ya que es innegable que la universidad es un espacio físico y simbólico para la generación, obtención y transferencia de aprendizajes diversos. Entre ellos, los saberes que motivan a los individuos a prepararse permanentemente (aprendizaje para toda la vida) sobre todo, los mediados por las Tecnologías de la Información y la Comunicación (TIC).

En ese sentido, el artículo discute los hallazgos del trabajo empírico realizado sobre las competencias de los universitarios para gestionar los medios y las TIC en un espacio escolar, con el interés de generar, a partir del diagnóstico, futuras propuestas de intervención para elevar mencionados saberes, ya que se parte de la idea de que la evaluación educativa es valiosa porque orienta hacia mecanismos de acción para transformar los contextos micro y macro educativos, sobre todo, ante las exigencias de los tiempos actuales en materia de digitalidad, la urgencia del pensamiento crítico y la participación en la denominada era Post-Web (O'reilly, 2005).

En la primera parte del texto se discuten las diferencias entre los conceptos competencia mediática y competencia digital, para explicar la categoría teórico-metodológica analizada en el artículo (creación y manipulación de contenido digital). Posteriormente se presentan las pautas y procedimientos que dan lugar a la estructura y sentido de los resultados.

\section{COMPETENCIAS MEDIÁTICAS Y DIGITALES EN LA EDUCACIÓN SUPERIOR}

El término competencia utilizado en las últimas décadas dentro del campo educativo refiere, según Díaz-Barriga (2006), a una suerte de discurso innovador sobre las reformas educativas implementadas en México y América Latina desde los años noventa y que, sus antecedentes provienen del campo de la lingüística y el mundo del trabajo. La primera acepción describe el concepto de competencia lingüística en alusión a un conjunto de saberes de la disciplina; la segunda, a las competencias de carácter utilitario del mundo empresarial como tareas, etapas, destrezas y habilidades de los sujetos para gestionar, desarrollar y vivir en el mundo laboral.

En tal, la noción de competencias dentro del currículum escolar nos remite a un escenario problemático, tal como argumenta Coll (2007), sin embargo, la falta de consenso permite develar diversas acciones orientadas a la construcción de ciudadanías empoderadas tecnológicamente.

Por lo anterior, la relevancia que las tecnologías y los medios han ganado en los procesos educativos formales, no formales e informales es muy amplia. No se puede negar que tales artefactos comunicativos permean la vida cotidiana de los sujetos en múltiples aspectos; socialización, aprendizaje y formas de insertarse en la política, la cultura y la economía. De ahí que, organismos a nivel global como la UNESCO (1982) desde la Declaración de Grünwald, el Instituto Nacional de Tecnologías Educativas y de Formación del Profesorado (INTEF, 2017) mediante su 
visión 2025 o incluso la Secretaría de Educación Pública en México (SEP, 2016) a través de los planes y programas de estudio, generen iniciativas para motivar cambios a los currículums escolares en todos los niveles, buscando alcanzar condiciones socioeducativas y de infraestructura pertinentes para la formación de los estudiantes respecto a las TIC.

Para la Asociación Nacional de Universidades e Instituciones de Educación Superior (ANUIES) las TIC "tienen un papel muy importante en el desarrollo de las sociedades, más cuando los cambios acelerados generan situaciones emergentes que conllevan a nuevas problemáticas que se convierten en áreas de oportunidad y en nuevos retos por resolver" (ANUIES, 2017, p.15). Asimismo, advierte que las Instituciones de Educación Superior (IES) deben priorizar sus esfuerzos en tres sentidos: 1) el gobierno de las TIC, 2) la ciberseguridad, y 3) la gestión interinstitucional con proveedores y prestadores de servicios tecnológicos, ello hacia la búsqueda de estrategias comunes para enfrentar los cambios socioeconómicos, culturales y educativos que devienen de la explosición tecnológica reciente.

Si bien en México la incorporación del lenguaje en competencias se introduce al currículum de manera sistemática a principios del año 2000, aún se carece de referenciales que hagan alusión a los conceptos competencia mediática y competencia digital, es decir, no hay propuestas articuladas que les integren a la narrativa educativa por lo que, la educación mediática y/o alfabetización informacional no se considera seriamente en las políticas educativas de la región. Los esfuerzos, regularmente, se dirigen a la integración de las TIC priorizando el uso de ordenadores y desatendiendo algunas recomendaciones hechas por la UNESCO desde hace más de 30 años en la Declaración de Grünwald que, marcaban la incorporación de los medios de comunicación al currículum escolar.

Para Aguaded-Gómez (2011) la educación mediática es un movimiento internacional imparable y una responsabilidad del sistema educativo, de los padres de familia y de diversos actores sociales puesto que, en espacios democráticas los medios deben contribuir a organizar formas de participación hacia ciudadanías críticas, responsables e igualitarias; objetivos que también se buscan desde la competencia mediática (CM) y la competencia digital (CD). La CM, hace referencia "al desarrollo de capacidades suficientes para interactuar con los medios de forma crítica y creativa: sea consumiento o produciendo contenidos" (Mateus, 2018, p.1).

Ferrés y Piscitelli (2012) realizan un esfuerzo teórico-metodológico de gran calado para proponer una taxonomía conceptual de la mano de investigadores internacionales y clarificar el término competencias mediáticas además, generar indicadores para medirles, clasificarles y cualificarles. Plantean seis dimensiones: 1) lenguajes, 2) tecnología, 3) procesos de interacción, 4) procesos de producción y difusión, 5) ideología y valores y 6) estética. Todas orientadas a la producción, gestión y análisis de contenidos mediáticos con y desde diversos entornos que propicien el pensamiento crítico.

Por otro lado, la competencia digital para González, Román y Prendes (2018) "entraña el uso seguro y crítico de las tecnologías de la sociedad de la información (TSI) para el trabajo, el ocio y la comunicación" (p.2). El Parlamento Europeo considera necesario motivar la adquisición de la 
competencia digital (INTEF, 2017) ya que es una de las 8 competencias clave que cualquier joven debe poseer al concluir la educación básica, ya que le permitirá desarrollar aprendizajes para toda la vida. Su propuesta se articula en 5 áreas: 1) información y alfabetización informacional 2) comunicación y colaboración 3) creación de contenidos digitales, 4) seguridad y 5) resolución de problemas.

Si bien los autores se refieren a la ciudadanía en general, las premisas cobran especial fuerza cuando se trabajan desde los contextos escolares puesto que, es en estos espacios donde se forjan iniciativas para dotar a los sujetos de las competencias. Para Grijalva-Verdugo y Moreno-Candil (2017) educar en medios implica un cambio de actitud docente para la incorporación de la tecnología a las estrategias pedagógicas y a la evaluación de los saberes digitales.

Consideramos que ambas conceptualizaciones son muy valiosas, puesto que no solo agrupan esfuerzos intelectuales hacia la educación mediática, sino que son complementarias. Sus indicadores además, son flexibles a los contextos culturales y educativos de cada región. Ya que las condiciones europeas (donde surgen los estudios), serán distintas a las que se viven en América Latina, o específicamente en México (lugar de esta investigación). Por lo que se retoman ambas para referirnos a un campo semántico que busca la promoción de saberes mediáticos y digitales en una era tan hipercomunicada (Lipovetsky y Serroy, 2009).

A partir de las conceptualizaciones anteriores, surge la propuesta de Ramírez-Martinell y Casillas (2016) denominada saberes digitales, misma que hace referencia a las capacidades de los sujetos para desenvolverse en el mundo digital, específicamente en el contexto de la educación superior puesto que, desde el espacio universitario se potencian acciones pedagógicas que dotan a los sujetos de saberes para forjar ciudadanías globales. En tal, poseer competencias mediáticas y digitales es una necesidad social, tal como argumentan Grijalva-Verdugo y Moreno-Candil (2016), Pérez-Ortega (2016), Ferrés-Prats, Aguaded-Gómez, y García-Matilla (2012).

En ese sentido, Ramírez-Martinell, Morales-Rodríguez y Olguín-Aguilar (2015) plantean una propuesta que sirve para categorizar los conocimientos que los universitarios deben poseer y que, adaptamos para evaluarles en el contexto de la IES propuesta (UAdeO). Para ello, se retomó la categoría analítica creación y manipulación de contenido digital, en alusión de las habilidades estudiantiles para el manejo de software, dominio de lenguajes digitales, manipulación de archivos de texto, gestión de conjuntos de datos, realización de videos y productos audiovisuales, entre otras.

\section{METODOLOGÍA}

El estudio puede catalogarse como descriptivo y correlacional. Para fines de operacionalización de las variables se ha dividido la dimensión creación y manipulación de contenido digital en tres subcategorías: 1) manipulación de archivos de texto, 2) manipulación de conjunto de datos y 3) manipulación de archivos multimedia, en atención a las propuestas de los autores. El cuestionario original consta de 60 ítems; 11 de ellos son los que apuntan al constructo investigativo propuesto. 
El instrumento de recolección de datos condensado (11 ítems) arroja un Alfa de Cronbach de .823 lo que representa un nivel de confianza alto. La validación del instrumento se realizó con el apoyo de cinco expertos (Red Temática Literacidad Digital en la Universidad), quienes seleccionaron los ítems que, a su criterio, son esenciales para la evaluación de los saberes del objeto de estudio, quedando con la siguiente estructura:

1. Sé cómo crear, abrir, guardar y editar archivos de texto, en programas como Word.

2. Sé cómo crear, abrir, guardar y editar hojas de cálculo, en programas como Excel.

3. Sé cómo crear, abrir, guardar y editar presentaciones dinámicas en programas como PowerPoint.

4. Utilizo fórmulas en Excel como suma, resta, división, multiplicación, etc.

5. Identifico si un equipo celular es mejor que otro según sus características.

6. Elaboro mapas mentales con programas como CmapTools, Freemind, entre otros.

7. Utilizo motores de búsqueda especiales para consultar información como bases de datos (Google Escolar, EBSCO, etc.).

8. Elaboro trípticos con apoyo de programas como CorelDraw, PowerPoint, etc.

9. Conozco los pasos para realizar un video o presentación audiovisual para mis tareas o reuniones formales.

10. Conozco diversos programas y/o aplicaciones para producción de materiales multimedia.

11. Puedo manipular archivos multimedia que contengan fotografía, video o audio.

El instrumento fue aplicado a estudiantes universitarios de la UAdeO mediante un muestreo probabilístico aleatorio simple. Al momento del levantamiento de datos, la unidad académica contaba con 3472 alumnos distribuidos en 14 programas educativos (ANUIES, 2017). Para la selección de la muestra se consideró un nivel de confianza del 95\% y un margen de error del 5\%. Obtenida la muestra ( $n=346$ estudiantes) se procedió a hacer una distribución proporcional de acuerdo al número de sujetos por programa educativo.

\section{$n=\frac{\left(Z^{\wedge} 2\right) * N * p^{*} q}{}$ \\ $\left(e^{\wedge} 2\right)(N-1)+\left(Z^{\wedge} 2\right) * p^{*} q$}

$N=3472$ alumnos

$\mathrm{p}=.5$

$q=.5$

$Z=1.96$

$n=346$ alumnos

Se trabajó con el hipotético de que las competencias mediáticas y digitales están diferenciadas por área de conocimiento. Es decir, el perfil profesional incide en los niveles de las competencias. Así, la $\mathrm{H}_{\circ}$ y la $\mathrm{H}_{1}$ se expresan de la siguiente forma:

Hipótesis nula $\left(H_{\circ}\right): \mu_{1}=\mu_{2}=\ldots=\mu_{\mathrm{k}}$ las competencias mediáticas y digitales no están asociadas al área de conocimiento. 
Hipótesis alternativa $\left(\mathrm{H}_{1}\right)$ : las competencias mediáticas y digitales son diferenciadas en relación al área de conocimiento de los universitarios.

Para el procesamiento de datos se utilizó el programa estadístico SPSS versión 21 . El formato del cuestionario fue a cinco puntos, sin embargo, para este análisis se transformó a 3 donde 1 significó competencia baja, 2 competencia media y 3 competencia alta. Realizadas las pruebas de normalidad (Kolmogorov-Smirnov y Shapiro-Wilk) se encontró que los datos no se distribuían de manera uniforme, por lo que se procedió a la realización de pruebas estadísticas no paramétricas (Chi-Cuadrado, $\mathrm{X}^{2}$ ), la variable de cruce fue el área de conocimiento: 1) ciencias sociales y humanidades, que agrupa carreras como ciencias de la comunicación, psicología, derecho, gobierno y administración pública, entre otras, 2) ingeniería y tecnología; sistemas computacionales y las ingenierías en software e industrial y 3) ciencias económico-administrativas; mercadotecnia, administración de empresas, turismo y contaduría.

\section{RESULTADOS}

La dimensión analizada (creación y manipulación de contenido digital), agrupa ítems afines a los saberes de los estudiantes para trabajar de manera dinámica y cotidiana en 1) manipulación de archivos de texto, 2) conjunto de datos y 3) programas multimedia, conjuntamente evalúa las capacidades para producir, gestionar y utilizar estos saberes en ámbitos escolares y profesionales. Ya que, de acuerdo a la literatura del campo, manejar contenidos digitales representa uno de los pilares fundamentales en la formación universitaria como argumentan Ottenbreit-Leftwich, Sadik, Sendurur y Sendurur (2012), respecto a la incorporación de las TIC a la actividad docente, al igual que lo hacen Keengwe y Bhargava (2013) cuando se refieren al uso de la tecnología en aspectos formales de la educación y socialización de los agentes involucrados.

\section{Manipulación de archivos de texto}

Al preguntar a los estudiantes sobre sus conocimientos para crear, abrir, guardar y editar archivos de texto en programas como Word, se encuentran hallazgos favorables puesto que, el $23.4 \%$ de los sujetos tiene competencia media y $76.6 \%$ alta. En tal, al distribuirse los datos de manera similar, no se encuentran diferencias estadísticamente significativas al interior de los grupos a razón de la variable de cruce (área de conocimientos) $X^{2}(2, N=346)=0.84, p<.05$ ).

Tabla 1. Sabe crear, abrir, guardar y editar archivos de texto en programas como Word

\begin{tabular}{cccc}
\hline Variable de agrupación & $\begin{array}{c}\text { Competencia baja } \\
(\%)\end{array}$ & $\begin{array}{c}\text { Competencia media } \\
(\%)\end{array}$ & $\begin{array}{c}\text { Competencia alta } \\
(\%)\end{array}$ \\
\hline General & 0.0 & 23.4 & 76.6 \\
Área de conocimientos & & & \\
Ciencias sociales y humanidades & 0.0 & 22.9 & 77.1 \\
Ingeniería y Tecnología & 0.0 & 25.0 & 75.0 \\
Ciencias económico-administrativas & 0.0 & 23.9 & 76.1 \\
\hline
\end{tabular}


Sin embargo, al evaluar el uso de programas informáticos menos populares como Excel, se encuentra que no todos los estudiantes, divididos por áreas del conocimiento, obtienen puntuaciones tan altas, de hecho, la mayoría se ubican en competencia media (55.2\%). Es ciencias sociales y humanidades el área donde se encuentran los estudiantes con la competencia más baja (4.4\%), mientras que los mejores evaluados son los alumnos de ciencias económicoadministrativas (52.3\% competencia alta). Se perciben diferencias estadísticamente significativas $X^{2}(4, N=346)=12.139, p<.05$ desde la variable de agrupación (área de conocimientos), por lo que en este ítem la variable de cruce incide en el nivel de competencia para el manejo de este tipo de programas. Ver tabla 2.

Tabla 2. Sabe crear, abrir, guardar y editar hojas de cálculo en programas como Excel

\begin{tabular}{cccc}
\hline Variable de agrupación & $\begin{array}{c}\text { Competencia baja } \\
(\%)\end{array}$ & $\begin{array}{c}\text { Competencia media } \\
(\%)\end{array}$ & $\begin{array}{c}\text { Competencia alta } \\
(\%)\end{array}$ \\
\hline General & 2.9 & 55.2 & 41.9 \\
Área de conocimientos* & & & \\
Ciencias sociales y humanidades & 4.4 & 60.5 & 35.1 \\
$\quad$ Ingeniería y Tecnología & 0.0 & 50.0 & 50.0 \\
Ciencias económico-administrativas & 0.9 & 46.8 & 52.3 \\
\hline
\end{tabular}

*Diferencia estadísticamente significativa $p<.05$

El último de los ítems de la categoría corresponde al manejo de presentaciones dinámicas en programas como PowerPoint; los resultados son positivos ya que, el $71.1 \%$ de los individuos tiene competencia alta. Al ser los hallazgos tan similares en las tres opciones de respuesta, no se encuentran diferencias estadísticas desde el área de conocimientos $X^{2}(4, N=346)=3.061, p<.05$.

Tabla 3. Sabe crear, abrir, guardar y editar presentaciones dinámicas en programas como PowerPoint.

\begin{tabular}{cccc}
\hline Variable de agrupación & $\begin{array}{c}\text { Competencia baja } \\
(\%)\end{array}$ & $\begin{array}{c}\text { Competencia media } \\
(\%)\end{array}$ & $\begin{array}{c}\text { Competencia alta } \\
(\%)\end{array}$ \\
\hline General & 0.6 & 28.3 & 71.1 \\
Área de conocimientos & & & \\
Ciencias sociales y humanidades & 1.0 & 26.3 & 72.7 \\
$\quad$ Ingeniería y Tecnología & 0.0 & 37.5 & 62.5 \\
Ciencias económico-administrativas & 0.0 & 29.4 & 70.6 \\
\hline
\end{tabular}

\section{Manipulación de conjunto de datos}

De acuerdo con Ramírez-Martinell y Casillas (2016), la manipulación de conjunto de datos tiene que ver con los conocimientos y habilidades de los estudiantes para identificar elementos operativos de un programa de procesamiento de datos (celdas, filas, columnas, registros). Por ejemplo, conocer las operaciones básicas de suma, resta, división y multiplicación para la gestión y filtrado de la información. 
Tabla 4. Utiliza fórmulas en Excel como suma, resta, división y multiplicación.

\begin{tabular}{cccc}
\hline Variable de agrupación & $\begin{array}{c}\text { Competencia baja } \\
(\%)\end{array}$ & $\begin{array}{c}\text { Competencia media } \\
(\%)\end{array}$ & $\begin{array}{c}\text { Competencia alta } \\
(\%)\end{array}$ \\
\hline General & 4.3 & 60.1 & 35.5 \\
Área de conocimientos* & & & \\
Ciencias sociales y humanidades & 7.3 & 67.8 & 24.9 \\
$\quad$ Ingeniería y Tecnología & 0.0 & 46.9 & 53.1 \\
Ciencias económico-administrativas & 0.0 & 49.5 & 50.5 \\
\hline
\end{tabular}

*Diferencia estadísticamente significativa $\mathrm{p}<.05$

En la tabla cuatro, se detallan diferencias estadísticamente significativas $X^{2}(4, N=346)=31.457$, $p<.05$ por área de conocimientos; siendo los estudiantes de ciencias sociales y humanidades los peor evaluados con apenas un $24.9 \%$ de competencia alta, frente a sus pares de las ingenierías y ciencias administrativas que obtienen el $53.1 \%$ y $50.5 \%$ respectivamente.

Otra de las herramientas importantes en la era digital, es el uso de programas para la creación de mapas mentales y de análisis para la vida escolar puesto que, estudios como los de Novak y Cañas (2005) señalan la relevancia del autoaprendizaje para la construcción de saberes permanentes o, lo que Delors (1996) denomina, aprendizaje para toda la vida. En ese sentido, se evaluaron los dominios de los jóvenes en relación a los programas digitales CmapTools y Freemind, donde se encuentran áreas de oportunidad importantes al respecto ya que, el 34.1\% de los jóvenes tiene competencia baja y apenas el $9.2 \%$ competencia alta.

Tabla 5. Elabora mapas mentales con programas como CmapTools, Freemind, entre otros

\begin{tabular}{cccc}
\hline Variable de agrupación & $\begin{array}{c}\text { Competencia baja } \\
(\%)\end{array}$ & $\begin{array}{c}\text { Competencia media } \\
(\%)\end{array}$ & $\begin{array}{c}\text { Competencia alta } \\
(\%)\end{array}$ \\
\hline General & 34.1 & 56.6 & 9.2 \\
$\quad$ Área de conocimientos* & & & 5.4 \\
Ciencias sociales y humanidades & 38.0 & 56.6 & 31.3 \\
$\quad$ Ingeniería y Tecnología & 12.5 & 56.3 & 10.1 \\
Ciencias económico-administrativas & 33.0 & 56.9 & \\
\hline
\end{tabular}

*Diferencia estadísticamente significativa $p<.05$

Al examinar los datos desde la variable de cruce se perciben diferencias estadísticas $X^{2}$ (4, $N=346)=25.528, p<.05$ ) debido a que hay distancias evidentes intergrupos, siendo los estudiantes de ingeniería y tecnología los que obtienen los porcentajes más altos (31.3\% competencia alta) y los de ciencias sociales y humanidades los más bajos (5.4\%).

Otro de los requerimientos de la cultura digital tiene que ver con la gestión y búsqueda de grandes cantidades de datos, así como con la identificación de textos especializados en el área de conocimiento de cada profesión por lo que, se evaluaron sus competencias para el manejo de motores de búsqueda y bases de datos como EBSCO, Scholar Google, entre otros. 
Los hallazgos dan cuenta que los individuos del área de ingeniería y tecnología poseen saberes más altos con un $62.5 \%$, seguidos de ciencias económico-administrativas $48.6 \%$. En ese sentido, los datos generales también son positivos dado que, el $46.8 \%$ de los sujetos se ubica en competencia media y alta; así, al ser los resultados empíricos tan similares no se encuentran diferencias estadísticamente significativas a razón de la variable de cruce (área de conocimientos) $X^{2}(4$, $N=346=5.881, p<.05$. Ver tabla 6 .

Tabla 6. Utiliza motores de búsqueda especializados para consultar información (Google escolar, EBSCO, entre otros.)

\begin{tabular}{cccc}
\hline Variable de agrupación & $\begin{array}{c}\text { Competencia baja } \\
(\%)\end{array}$ & $\begin{array}{c}\text { Competencia media } \\
(\%)\end{array}$ & $\begin{array}{c}\text { Competencia alta } \\
(\%)\end{array}$ \\
General & 6.4 & 46.8 & 46.8 \\
Área de conocimientos & & & \\
Ciencias sociales y humanidades & 7.8 & 48.8 & 43.4 \\
Ingeniería y Tecnología & 0.0 & 37.5 & 62.5 \\
Ciencias económico-administrativas & 5.5 & 45.9 & 48.6 \\
\hline
\end{tabular}

\section{Manipulación de archivos multimedia}

En esta categoría se analizan los saberes para la producción, reproducción, edición e integración de medios en un producto multimedia, así como su distribución en diversos soportes digitales (Ramírez-Martinell y Casillas, 2016).

El primer ítem tiene que ver con la capacidad de los estudiantes para distinguir las características de Hardware y Software en dispositivos móviles, debido a que su penetración en los ámbitos educativos, sociales y culturales ha crecido de manera acelerada en los últimos años, tal como declara el estudio IAB México (Interactive Advertising Bureau, 2017) donde se encuentra que 71.5 millones de mexicanos son internautas, equivalente al 60\% de la población; de los cuales el $88 \%$ tiene un Smartphone.

Si bien, el estudio no cuestiona las posibilidades del móvil en el aula universitaria, explora los conocimientos para identificar sus características, tal como, capacidades de almacenamiento, velocidad de navegación, tipo de sistema operativo, aplicaciones de mayor uso, entre otras.

La competencia alta es la dominante; $54.0 \%$, seguida de competencia media; 44.5\%. A pesar de que los saberes son altos, se observa que son más positivos en los sujetos de ingeniería y tecnología (81.2\% competencia alta), tal como podía esperarse debido al currículum escolar donde están matriculados, razón por la que al realizar las pruebas de hipótesis se encuentran diferencias entre los grupos $X^{2}(4, N=346)=12.563, p<.05$. Tabla 7 . 
Tabla 7. Identifica si un equipo celular es mejor que otro según sus características

\begin{tabular}{cccc}
\hline Variable de agrupación & $\begin{array}{c}\text { Competencia baja } \\
(\%)\end{array}$ & $\begin{array}{c}\text { Competencia media } \\
\text { General }\end{array}$ & $\begin{array}{c}\text { Competencia alta } \\
\text { (\%) }\end{array}$ \\
Área de conocimientos* & 1.4 & 44.5 & 54.0 \\
Ciencias sociales y humanidades & 1.5 & & \\
Ingeniería y Tecnología & 0.0 & 44.4 & 54.1 \\
Ciencias económico-administrativas & 1.8 & 18.8 & 81.2 \\
\hline
\end{tabular}

*Diferencia estadísticamente significativa $\mathrm{p}<.05$

Por otro lado, la tabla 8 refleja las habilidades de los jóvenes para el manejo de paquetería concerniente al diseño de materiales creativos necesarios para exposiciones académicas y/o el mundo del trabajo. Los hallazgos dan cuenta de competencias similares en todas las áreas de conocimiento con predominancia en la competencia media; $56.4 \%$. Así, al realizar las pruebas de Chi no se encuentran diferencias $X^{2}(4, N=346)=1.886, p<.05$ entre los grupos.

Tabla 8. Elabora trípticos con apoyo de programas como CorelDraw, PowerPoint, etc.

\begin{tabular}{cccc}
\hline Variable de agrupación & $\begin{array}{c}\text { Competencia baja } \\
(\%)\end{array}$ & $\begin{array}{c}\text { Competencia media } \\
(\%)\end{array}$ & $\begin{array}{c}\text { Competencia alta } \\
(\%)\end{array}$ \\
\hline General & 6.6 & 56.4 & 37.0 \\
Área de conocimientos & & & \\
Ciencias sociales y humanidades & 5.9 & 56.1 & 38.0 \\
Ingeniería y Tecnología & 6.3 & 50.0 & 43.2 \\
Ciencias económico-administrativas & 8.3 & 58.7 & 33.0 \\
\hline
\end{tabular}

Mucho se ha dicho en la literatura educativa y tecnológica sobre la importancia de realizar materiales audiovisuales como parte de los procesos formativos para los ciudadanos del siglo XXI ya que, predomina el hipotético de que las generaciones jóvenes requieren de experiencias sensoriales a través de la música, la imagen y el movimiento para construir aprendizajes significativos. La tecnología digital ha resaltado la importancia de su utilización en las aulas. No se trata solo de una cuestión tecnológica sino de una dimensión metodológica, pedagógica e ideológica (Aparici, 2011, p.6).

En lo anterior, es importante que los universitarios conozcan los pasos para realizar un video o presentación audiovisual con fines escolares, ello posibilitará el registro y gestión de recursos multimedia en procesos interactivos diversos. De este modo, se encuentra que los estudiantes de ciencias sociales y humanidades poseen saberes más altos (38.5\%), seguidos de ingeniería y tecnología (31.3\%) y ciencias económico administrativas (27.5\%). Al distribuirse los datos de manera tan uniforme en el espectro de respuestas no se encuentran diferencias estadísticamente significativas desde la variable de contraste $\left.X^{2}(4, N=346)=4.761, p<.05\right)$. Vera tabla 9. 
Tabla 9. Conoce los pasos para realizar un video o presentación audiovisual para tareas o exposiciones formales.

\begin{tabular}{cccc}
\hline Variable de agrupación & $\begin{array}{c}\text { Competencia baja } \\
(\%)\end{array}$ & $\begin{array}{c}\text { Competencia media } \\
(\%)\end{array}$ & $\begin{array}{c}\text { Competencia alta } \\
(\%)\end{array}$ \\
\hline General & 5.8 & 59.8 & 34.4 \\
Área de conocimientos & & & \\
Ciencias sociales y humanidades & 4.9 & 56.6 & 38.5 \\
$\quad$ Ingeniería y Tecnología & 9.4 & 59.4 & 31.3 \\
Ciencias económico-administrativas & 6.4 & 66.1 & 27.5 \\
\hline
\end{tabular}

Otro de los ítems de la dimensión tiene que ver con el conocimiento de programas informáticos para producir materiales multimedia, en el entendido que estos saberes son valiosos en la era de las pantallas. Se descubre que el promedio de los estudiantes, se ubican en competencia media con un 63\%, competencia alta con el 33.5\% y 3.5\% competencia baja. Los análisis desde la variable de cruce arrojan diferencias estadísticamente significativas $X^{2}(4, N=346)=12.396, p<.05$ y es que, son los estudiantes de ciencias sociales y humanidades quienes presentan mayores dominios en ese sentido. Ver tabla 10.

Tabla 10. Conoce diversos programas y aplicaciones digitales para producción de materiales multimedia

\begin{tabular}{cccc}
\hline Variable de agrupación & $\begin{array}{c}\text { Competencia baja } \\
(\%)\end{array}$ & $\begin{array}{c}\text { Competencia media } \\
(\%)\end{array}$ & $\begin{array}{c}\text { Competencia alta } \\
(\%)\end{array}$ \\
\hline General & 3,5 & 63,0 & 33,5 \\
Área de conocimientos* & & & \\
Ciencias sociales y humanidades & 1,5 & 60,5 & 38,0 \\
Ingeniería y Tecnología & 3,1 & 59,4 & 37,5 \\
Ciencias económico-administrativas & 7,3 & 68,8 & 23,9 \\
\hline
\end{tabular}

*Diferencia estadísticamente significativa $\mathrm{p}<.05$

De igual manera, al evaluar capacidades más técnicas para la producción de materiales audiovisuales, son los jóvenes de ingeniería y tecnología quienes consiguen las puntuaciones más elevadas; $65.6 \%$ (competencia alta), seguidos de ciencias sociales y humanidades; $36.1 \%$ y ciencias económico-administrativas; $30.3 \%$. Así, tales puntajes son congruentes con las pruebas estadísticas donde el valor de $p$ es inferior a $.05 X^{2}(4, N=346)=12.396, p<.05$. Ver tabla 11.

Tabla 11. Puede manipular archivos multimedia con contenidos de fotografía, video y audio

\begin{tabular}{cccc}
\hline Variable de agrupación & $\begin{array}{c}\text { Competencia baja } \\
(\%)\end{array}$ & $\begin{array}{c}\text { Competencia media } \\
(\%)\end{array}$ & $\begin{array}{c}\text { Competencia alta } \\
(\%)\end{array}$ \\
\hline General & 4.0 & 59.0 & 37.0 \\
Área de conocimientos* & & & \\
Ciencias sociales y humanidades & 2.4 & 61.5 & 36.1 \\
$\quad$ Ingeniería y Tecnología & 3.1 & 31.3 & 65.6 \\
Ciencias económico-administrativas & 7.3 & 62.4 & 30.3 \\
\hline
\end{tabular}

*Diferencia estadísticamente significativa $\mathrm{p}<.05$ 
Los resultados de la tabla 11 son interesantes debido a que, los estudiantes de las ingenierías y carreras tecnológicas son quienes poseen saberes más destacados en asuntos técnicos para la manipulación de archivos audiovisuales, sin embargo, las tablas 9 y 10 dan cuenta de que los alumnos de ciencias sociales son quienes salen mejor posicionados en asuntos creativos para la producción multimedia, es decir, en el conocimiento de programas y pasos para la producción.

\section{CONCLUSIONES}

Se encuentra que el área de conocimiento de los universitarios incide de manera parcial en sus niveles de competencia mediática puesto que, de los 11 ítems el 54.54\% son estadísticamente significativos $(\mathrm{p}<.05)$, por lo tanto, se acepta la hipótesis del investigador $(\mathrm{H} 1)$.

Al analizar a detalle las respuestas de los universitarios se identifican áreas de oportunidad en el manejo de conjunto de datos como Excel, CmapTools y Freemind, por lo que sería importante generar mecanismos de intervención educativa en ese sentido, al igual que en la producción de materiales multimedia y manipulación de los mismos, ya que una de las condiciones de la competencia mediática es que los ciudadanos cuenten con herramientas y estrategias para la gestión, análisis y consumo de productos digitales de diversa índole. Tal como recomiendan organismos a nivel global (UNESCO, Parlamento Europeo, SEP, etc.)e investigaciones como las de Gisbert y Esteve (2016), Dalton (2017), Hernández-Pérez y García-Moreno (2010).

En términos generales, los niveles de competencia mediática son positivos ya que al promediar los porcentajes de los 11 ítems que componen la dimensión manipulación y creación de contenido digital, se encuentra que el $6.35 \%$ de los estudiantes se ubica en competencia baja, el $50.28 \%$ en competencia media y el $43.37 \%$ en competencia alta. Tal panorama, permite vislumbrar indicadores desde dónde se pueden diseñar estrategias de intervención educativa para incrementar los saberes mediáticos y digitales, así como acciones institucionales que garanticen que los jóvenes adquieran las competencias durante su formación universitaria.

Por lo tanto, las competencias mediáticas y digitales deben integrarse oficialmente al currículo escolar ya que, las TIC y los medios están presentes en la vida diaria de los sujetos, por lo que se requieren investigaciones de mayor alcance que permitan cartografiar las prácticas socioeducativas de los universitarios y de diversos actores escolares: profesores, gestores y directivos, a fin de comprender de manera integral el fenómeno.

En lo anterior, los hallazgos generan preocupaciones que se están canalizando en nuevas líneas de investigación, sobre todo en lo concerniente con los profesores. Se ha iniciado con el diagnóstico de las CM y CD en varias universidades del país y desde ahí, se buscará diseñar e implementar estrategias pedagógicas que motiven la adquisición de los saberes en la región. 


\section{REFERENCIAS}

Aguaded-Gómez, I. (2011). La educación mediática, un movimiento internacional imparable. La ONU, Europa y España apuestan por la educomunicación. Comunicar, 19(37), 7-8.

Recuperado de http://www.redalyc.org/articulo.oa?id=15820024001

ANUIES. (6 de octubre de 2017). Asociación Nacional de Universidades e Instituciones de Educación Superior. Recuperado de http://www.anuies.mx/noticias/las-tecnologas-de-la-informaciny-las-comunicaciones-factor

Aparici, R. (2011). Principios pedagógicos y comunicacionales de la educación 2.0. La educación digital magazine, 145, 1-14. Recuperado de http://www.educoas.org/portal/La Educacion Digital/laeducacion 145/articles/Roberto Aparici.pdf

Dalton, E. M. (2017). Beyond Universal Design for Learning: Guiding Principles to Reduce Barriers to Digital \& Media Literacy Competence. Journal of Media Literacy Education, 9(2), 17-29. Recuperado de https://eric.ed.gov/?id=EJ1160465

González, V., Román, M., y Prendes, M. (2018). Formación en competencias digitales para estudiantes universitarios basada en el modelo DigComp. Edutec. Revista Electrónica de Tecnología Educativa, (65), 1-15. Recuperado de http://www.edutec.es/revista/index.php/edutece/article/view/1119

Gisbert, M., y Esteve, F. (2016). Digital Leaners: la competencia digital de los estudiantes universitarios. La cuestión universitaria, (7), 48-59. Recuperado de http://polired.upm.es/index.php/lacuestionuniversitaria/article/view/3359

Coll, C. (2007). Las competencias en la educación escolar: algo más que una moda y mucho menos que un remedio. Aula de innovación educativa,161, 34-39. Recuperado de http://www.vila.com.br/html/outros/2010/30_anos/pdf_30/07\%20Cesar.pdf

Delors, J. (1996). La educación encierra un tesoro. París: UNESCO/Santillana.

Díaz-Barriga, Á. (2006). El enfoque de competencias en la educación:¿ Una alternativa o un disfraz de cambio?.Perfiles educativos, 28(111), 7-36. Recuperado de http://www.redalyc.org/articulo.oa?id=13211102

Ertmer, P., Ottenbreit-Leftwich, A., Sadik, O., Sendurur, E., \& Sendurur, P. (2012). Teacher beliefs and technology integration practices: A critical relationship. Computers \& Education(59), 423-435. Recuperado de https://www.sciencedirect.com/science/article/pii/S0360131512000437 
Ferrés Prats, J., Aguaded-Gómez, I., y García-Matilla, A. (2012). La competencia mediática de la ciudadanía española competencias y retos. Icono 14, 10(2), 23-42. Recuperado de https://icono14.net/ojs/index.php/icono14/article/view/201

Ferrés, J., y Piscitelli, A. (2012). La competencia mediática: propuesta articulada de dimensiones e indicadores [Media Competence. Articulated Proposal of Dimensions and Indicators]. Comunicar, (38), 75-82. Recuperado de https://doi.org/10.3916/C38-2012-02-08.

Grijalva-Verdugo, A., \& Moreno-Candil, D. (2017). Empoderamiento social en contextos violentos mexicanos mediante la competencia mediática. Comunicar, 25(53), 29-38.

Recuperado de https://revistacomunicar.wordpress.com/2018/01/11/empoderamientosocial-en-contextos-violentos-mexicanos-mediante-la-competencia-mediatica-2/

Grijalva-Verdugo, A., y Moreno-Candil, D. (2016). Competencia mediática en jóvenes universitarios: análisis socioeducativo del currículum escolar. Sociología y Tecnociencia, 1(6), 14-25. Recuperado de https://revistas.uva.es/index.php/sociotecno/article/view/652

Hernández-Pérez, T., \& García Moreno, M. A. (2010). Los retos de la alfabetización informacional en las bibliotecas: guía para superar la brecha entre nativos e inmigrantes digitales. Revista Prisma. Com, (13). Recuperado de https://eprints.ucm.es/12139/

IAB México. (2017). Estudio de Consumo de Medios y Dispositivos entre Internautas Mexicanos 2016. Interactive Advertising Bureau. Ciudad de México: MillwardBrown.

INTEF. (2017). Marco Común de Competencia Digital Docente . Instituto Nacional de Tecnologías Educativas y de Formación del Profesorado. Ministerio de Educación de Cultura y Deporte e Instituto Nacional de Tecnologías Educativas y de Formación del Profesorado .

Keengwe, J., \& Bhargava, M. (2014). Mobile learning and integration of mobile technologies in education. Education and Information Technologies, 19(4), 737-746. Recuperado de https://link.springer.com/article/10.1007/s10639-013-9250-3

Lipovetsky, G., y Serroy, J. (2009). La pantalla global. Cultura mediática y cine en la era hipermoderna. Barcelona: Anagrama.

Mateus, J. (2018). EDUCARED. Obtenido de Educación mediática aprender y enseñar en un mundo digital : Recuperado de http://educared.fundaciontelefonica.com.pe/educacionmediatica/

Novak, J., y Cañas, A. (2005). Construyendo sobre Nuevas Ideas Constructivistas y la Herramienta CmapTools para Crear un Nuevo Modelo para Educación. Eduteka. Recuperado de http://www. eduteka. org/pdfdir/CmapToolsNuevoModeloEducacion. pdf. 
O'reilly, T. (2005). What is Web 2.0? Design patterns and bussines models for the next generation of software. En H. Donelan, K. Kear, y M. Ramage, Online Communication and Colaboration: A reader, 225-289. Oxon: Routledge Taylor y Francis Group.

Pérez-Ortega, I. (2016). La competencia mediática en el currículo escolar: ¿espacio para innovaciones educativas con tecnologías de la información y la comunicación? [Media competency in academic programs: Is there space for educational innovations with information and communication technology?]. Innovación educativa , 16(70), 61-84.

Ramírez-Martinell, A., y Casillas, M. (marzo de 2016). Los saberes digitales y las disciplinas universitarias. Recuperado de www.uv.mx/blogs/brechadigital

Ramírez-Martinell, A., Morales-Rodríguez, A., y Olguín-Aguilar, P. (2015). Marcos de referencia de saberes digitales. EDMETIC, 4(2), 112-136.

SEP. (2016). El modelo educativo 2016 el planteamiento pedagógico de la Reforma Educativa. Ciudad de México: Secretaria de Educación Pública.

UNESCO. (1982). Declaración de Grünwald sobre la educación relativa a los medios de comunicación. Grünwald, Alemania: UNESCO

\section{Para referenciar este artículo:}

Grijalva Verdugo, A., \& Lara Rivera, J. (2019). Competencias mediáticas en jóvenes universitarios. Análisis de saberes para producir contenido digital en una IES mexicana. Edutec. Revista Electrónica De Tecnología Educativa, (67). https://doi.org/10.21556/edutec.2019.67.1297 